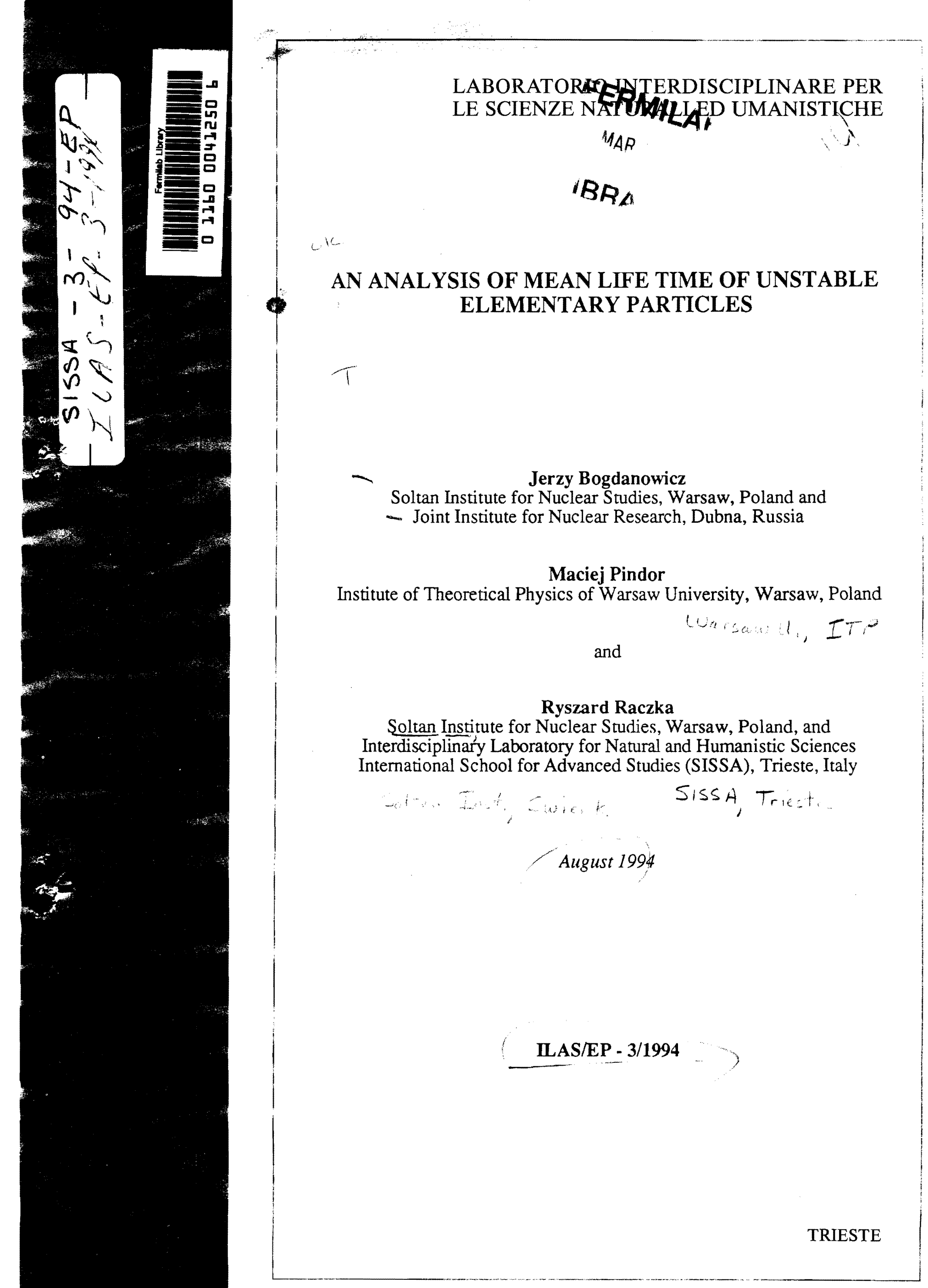




\title{
AN ANALYSIS OF MEAN LIFE AND LIFE TIME OF UNSTABLE ELEMENTARY PARTICLES
}

\author{
Jerzy Bogdanowicz \\ Soltan Institute for Nuclear Studies, Warsaw, Poland, and \\ Joint Institute for Nuclear Research, Dubna, Russia
}

\begin{abstract}
Maciej Pindor
Institute of Theoretical Physics of Warsaw University, Warsaw, Poland

and

Ryszard Rączka ${ }^{1,2}$

Soltan Institute for Nuclear Studies, Warsaw, Poland, and Interdisciplinary Laboratory for Natural and Humanistic Sciences International School for Advanced Studies (SISSA), Trieste, Italy
\end{abstract}

August 1994

\begin{abstract}
The theoretical analysis of a concept of life time and mean life of unstable elementary particles is presented. The new analytic formulas for life time and mean life as the function of decay width $\Gamma$ and the mass of unstable particle are derived for Breit-Wigner and Matthews-Salam energy distributions. It is demonstrated that for unstable particles with a larger width or decay energy threshold the deviation from the generally accepted mean life $\tau^{\mathrm{m}}=\Gamma^{-1}$ is significant. The behaviour of the decay law $\mathrm{P}(\mathrm{t})$ for small times is analyzed and it is shown that the Breit-Wigner distribution violates the condition $\dot{P}(t=0)=0$ whereas the Matthews-Salam distribution satisfies it.
\end{abstract}

\footnotetext{
${ }^{1}$ e-mail: RRACZKA@fuw.edu.pl

${ }^{2}$ Partially supported by the Stiftung Für Deutsch-Polnische Zusammenarbeit Grant N. $984 / 94 / \mathrm{LN}$
} 


\section{Introduction}

Almost all elementary particles are unstable [1]. However the instability effects are usually disregarded in a description of elementary particle interactions. In fact in most of applications of Standard Model one uses, as a main tool, the S-matrix approach in which one assumes implicitly or explicitly that asymptotic states exist also for unstable particles such as $\mu, \tau, \mathrm{Z}^{0}, \mathrm{~W}^{ \pm}$and other elementary particles. On the other hand analyzing the energy dependence of various cross sections like e.g. the total cross section $\mathrm{e}^{+}+\mathrm{e}^{-} \rightarrow$ hadrons we observe the striking effects due to instabilities of exchanged vector mesons like $\rho, \omega, \phi, \mathrm{J} / \Psi, \Upsilon$ and $\mathrm{Z}[1]$.

We have decided therefore to investigate in details the properties of unstable particles. We present in this work the detailed analysis of the concept of life time, and mean life as the function of decay width $\Gamma$, mass $m$ and the threshold energy $\lambda_{\mathrm{tr}}$, since these quantities give the important characteristics of unstable elementary particles.

In all of experimental analyses one identifies the mean life $\dot{\tau}_{\psi}^{\mathrm{m}}$ of unstable particle $\psi$ with the quantity

$$
\dot{\tau}_{*}^{m}=\frac{1}{\Gamma}
$$

where $\Gamma$ is the decay width [1]. This relation would be true if the decay law $\mathrm{P}_{\psi}(\mathrm{t})$ (defined below) would be given by pure exponential i.e.

$$
P_{\psi}(t)=\exp (-\Gamma t) \quad \text { for all } \quad t \geq 0
$$

It was observed however in several works that the decay law for unstable systems cannot be given by pure exponential. In fact we have:

Theorem 1.1. Let $\psi$ be a state of an unstable system and let $\mathrm{C}$ and $\mathrm{B}$ be some positive constants such that

$$
P_{t}(t) \leq C e^{-B t}
$$

for all $\mathrm{t} \geq 0$. Then the spectrum of the corresponding energy operator $H$ equals to $R=$ $(-\infty, \infty)$. (For the proof see [2], Thm. 1.5.7).

Since the energy spectrum of a physical system must be bounded from below the 
Theorem 1.1 states that the decay law $\mathrm{P}_{\psi}(\mathrm{t})$ cannot be given by pure exponential. Hence the formula (1.1) for mean life is incorrect.

The more detailed analysis indicates that we must have, in general, a deviation from pure exponential for small and large times: in particular we have ([2], [3])

$$
\left.\frac{d P_{t}(t)}{d t}\right|_{t=0}=0
$$

whereas for pure exponential this derivative equals $-\Gamma$; for large times we have

$$
P_{*}(t)=N\left[\exp (-\Gamma t)+c t^{-n}\right]
$$

with $n>1$ and $n$ and $c$ depending on the shape of energy spectrum. [2], [3], [4].

The analysis shows that the algebraic time dependence in (1.4) follows from the boundedness from below of energy operator and is therefore unavoidable. Consequently if the time of measurement increases the algebraic term becomes dominant and there is no reason to relate $\tau_{\psi}^{\mathrm{m}}$ with $\Gamma$ by formula (1.1). There arises therefore the need of a general definition of mean life in terms of decay law $\mathrm{P}_{\psi}(\mathrm{t})$.

In order to solve these problems we present in Sec. 2 the general definitions of life time $\tau_{\psi}$ and mean life $\tau_{\psi}^{\mathrm{m}}$ in terms of decay law $\mathrm{P}_{\psi}(\mathrm{t})$. We follow the conventions given in [2]. We show that from the fundamental properties of unstable particle with the width $\Gamma$ (to be defined later) and mass $m$ it follows that in general

$$
\tau_{*}^{m}=\frac{1}{\Gamma}[1+\Delta \tau(\Gamma / m)]
$$

where the analytic form of the additional term $\Delta \tau(\Gamma / \mathrm{m})$ depends on the form of energy distribution. For frequently utilized Breit-Wigner form of the energy distribution we find the analytic form of the function of $\Delta \tau(\Gamma / \mathrm{m})$ : if $\Gamma / \mathrm{m}$ is relatively large as it is for e.g. $\rho$ meson $(\Gamma / \mathrm{m} \approx 0.2)$ or $Z^{0}$-meson $(\Gamma / \mathrm{m} \simeq 0.02)$ then $\Delta \tau(\Gamma / \mathrm{m})$ is numerically significant and one must use (1.5) instead of (1.1) for the precise determination of $\tau_{\psi}^{\mathrm{m}}$.

We illustrate the above problems for the Breit-Wigner energy distribution applied to the analysis of mean life of $\rho$ - and $Z^{0}$-mesons. The numerical analysis clearly shows that mean life obtained from formula (1.1) and (1.5) are different. We also show the interesting result that the Breit-Wigner distribution violates the condition (1.3); this is a serious defect of $\mathrm{B}-\mathrm{W}$ distribution which leads to a question if $\mathrm{B}-\mathrm{W}$ distribution is 
meaningful in particle physics.

We analyze in Sec. 3 the above problems for the Matthews-Salam energy distribution, which is derived in the frame-work of the relativistic quantum field theory, and provides a convenient description of the relativistic unstable particles. We show that decay properties of relativistic particles are essentially different from that described by $\mathrm{B}-\mathrm{W}$ energy distributions. In particular we show that the M-S energy distribution satisfies the condition (1.3).

We conclude this work with a discussion of obtained results and with some suggestions for concrete experiments which could clarify the problem of a nature and properties of decay law for unstable particles. 


\section{Life time and mean life.}

Let $H$ be an energy operator with the spectral representation

$$
H=\int \lambda d E(\lambda)
$$

where $E(\lambda)$ is the spectral measure of $H$. Let $U_{t}$ be the evolution operator

$$
U_{t}=\exp (-i t H)=\int e^{-i t \lambda} d E(\lambda)
$$

and let $\psi$ be a unit vector in a Hilbert space $\#$ of physical states. Let $v_{\psi}(t)$ be the probability amplitude

$$
v_{\psi}(t)=\left(\psi, U_{t} \psi\right)
$$

Then the decay law $P_{\psi}(t)$ for the system described by the state vector $\Psi$ is given by the formula

$$
P_{\psi}(t)=\left|\nu_{\psi}(t)\right|^{2}=\left|\left(\psi, U_{t} \psi\right)\right|^{2}=\left|\int e^{-i \lambda t} d(\psi, E(\lambda) \psi)\right|^{2}
$$

The quantity $P_{\psi}(t)$ has the natural physical interpretation as the probability that the system in the state $\psi$ at $t=0$ will not decay till the next measurement carried out at the time $t>0$.

We now introduce two important characteristic of unstable particles namely the life-time $\tau$ and the mean life $\tau^{\mathrm{m}}[2]$.

The life time $\tau_{\psi}$ of an unstable particle represented at $t=0$ by the state $\psi$ is given by the formula

$$
\tau_{\psi}=\int_{0}^{\infty} P_{\psi}(t) d t
$$

The mean life $\tau_{\psi}{ }^{\mathrm{m}}$ is defined by the formula

$$
\tau_{\psi}^{m}=-\int_{0}^{\infty} t d P_{\psi}(t)
$$


Integration by parts gives:

$$
\tau_{\downarrow}^{m}=\tau_{\downarrow}-\lim _{t \rightarrow \infty} t P_{\downarrow}(t)
$$

For pure exponential decay law $P_{\phi}(t)=\exp (-\Gamma t)$ for all $t \geq 0$ we have:

$$
\tau_{*}^{m}=\tau_{*}
$$

However if $P_{\psi}(t)$ falls off for large $t$ as $c t^{1}$ we obtain

$$
\tau_{*}^{m}=\tau_{\downarrow}-c
$$

Hence in general the life time and mean life may be different.

We wish to stress that definitions (2.5) and (2.6) are the only possible definitions of mean life and life time which in case of pure exponential reduce to the well known formula

$$
\tau_{*}=\tau_{*}^{m}=\Gamma^{-1}
$$

It was first proven by Khalfin [4] that the exponentiality of a decay law of any unstable system - especially for very small and large times - must be violated. The descriptions of this phenomena was given in Theorem 1.1. Since for physical unstable systems the energy operator $H$ must be bounded from below the decay low $P_{\psi}(t)$ cannot be pure exponential i.e. $P_{\psi}(t) \neq \exp (-\Gamma t)$ for all $t \geq 0$.

We shall analyze in what follows the concept of mean life, since this quantity is mainly considered in various experiments with unstable particles [1]. It should be stressed that in most of experiments one reads off the mean life from a slope of exponential. However the Theorem 1.1 and Eqs. (1.3) and (1.4) indicate that the true decay law $P(t)$ must be different from the exponential decay law.

We now give for an illustration the analysis of mean life for the Breit-Wigner distribution of energy. In this case we have [2]

$$
d(\Psi, E(\lambda) \psi)=g(\lambda) d \lambda
$$

with 


$$
g(\lambda)=\frac{\Gamma N}{2 \pi} \frac{\theta\left(\lambda-\lambda_{t r}\right)}{(\lambda-m)^{2}+\frac{1}{4} \Gamma^{2}}
$$

and

$$
N=\left[\frac{1}{2}+\pi^{-1} \operatorname{arctg}\left(\frac{2 \tilde{m}}{\Gamma}\right)\right]^{-1}
$$

where $\lambda_{\mathrm{tr}}$ is the threshold energy for the decaying particle, $\widetilde{\mathrm{m}}=\mathrm{m}-\lambda_{\mathrm{tr}}$ and $\theta$ is, the Heaviside's function. We note that it is (2.11) and later (3.1) which gives the precise meaning to $\Gamma$.

We observe that if $\Gamma \ll \widetilde{\mathrm{m}}$ then $N$ is almost equal to one.

Using (2.3) one obtains [2]

$$
\psi(t)=N\left[\exp \left(-i \tilde{m} t-\frac{1}{2} \Gamma t\right)-i(2 \pi)^{-1} \sum_{\alpha= \pm 1} \exp \left(-i \tilde{m} t-\frac{1}{2} \alpha \Gamma t\right) E i\left(i \tilde{m} t+\frac{1}{2} \alpha \Gamma t\right)\right]
$$

where $\operatorname{Ei}(z)$ is the integral exponential function.

For large $t$ we obtain

$$
v_{a s}(t)=N\left[\exp \left(-i \tilde{m} t-\frac{1}{2} \Gamma t\right)-i(2 \pi)^{-1} \frac{\Gamma}{\tilde{m}^{2}+\frac{1}{4} \Gamma^{2}} t^{-1}+0\left(t^{-2}\right)\right]
$$

We see that pure exponential decay is modified - for large times - by the algebraic one. Since for large times $\mathrm{P}_{\psi}(\mathrm{t})=\mathrm{c}_{1} \mathrm{t}^{-2}$ by Eq. (2.7) we have $\tau_{\psi}=\tau_{\psi}^{\mathrm{m}}$. Consequently we have four regions of time intervals:

I. The region of small times where using (2.13) we obtain:

$$
P_{\psi}(t)=1-\frac{N}{2} \Gamma t+(\Gamma t)^{2}\left[c_{0}+c_{1} \ln (\Gamma t)+c_{2} \ln ^{2}(\Gamma t)\right]+0\left(t^{3}\right)
$$

where $c_{1}$ are definite constants.

II. The region of intermediate times where the exponential term is dominating: in this region we have:

$$
P_{4}(t)-N^{2} \exp (-\Gamma t)+0\left(\frac{\exp (-\Gamma / 2 t)}{t}\right)
$$


III. The third region where the exponential term is comparable with the algebraic one. This occurs when modulus of exponential term is comparable with the modulus of the algebraic term i.e. for $t$ in the neighbourhood of $t_{c}$ determined from the formula

$$
\frac{1}{2} \Gamma t_{c} \exp \left(-\frac{1}{2} \Gamma t_{c}\right)=\pi^{-1}\left(\frac{\Gamma}{2 \tilde{m}}\right)^{2}
$$

IV. The fourth region for $t \gg t_{c}$ where the decay law is dominated by the algebraic term

$$
P_{\psi}(t) \propto c_{1} t^{-2}+0\left(t^{-3}\right)
$$

We note that from $(2.15)$ we get:

$$
d P_{*}(t) /\left.d t\right|_{t=0}=-N \Gamma / 2<0
$$

Hence the B-W distribution violates the condition (1.3).

Equation (2.17) has two solutions for $\Gamma t_{c}$. From the shape of the curve $x \exp (-x)$ it follows that (2.17) will have one solution near zero and the another one rather large. Since (2.17) is derived from the asymptotic expansion (2.14) only the large solution for $\Gamma t_{c}$ is meaningful. We see that if $\Gamma / \overline{\mathrm{m}}$ increases the solution $\Gamma \mathrm{t}_{\mathrm{c}}$ for $(2.17)$ decreases. Consequently for unstable particles with a larger width $\Gamma$ the function $\mathrm{P}_{\Psi}(\mathrm{t})$ will start to oscillate for smaller times. For instance for $\rho$-meson with $\Gamma=152 \mathrm{MeV}$ and $\tilde{\mathrm{m}}=490 \mathrm{MeV}$ we obtain $\Gamma t_{c}=13.58$ : for $Z^{0}$-meson with $\Gamma=2.487 \mathrm{GeV}, \overline{\mathbf{m}}=91,17 \mathrm{GeV}$ we obtain $\Gamma t_{c}=24.47$.

We present now the typical example of oscillation with time of the decay law $\mathrm{P}_{\psi}(\mathrm{t})$.

We took for an illustration $\Gamma=152 \mathrm{MeV}, \mathrm{m}=770 \mathrm{MeV}, \lambda_{\mathrm{tr}}=280 \mathrm{MeV}$ which corresponds to $\rho$-meson characteristic parameters.

The oscillations of $P_{\psi}(t)$ are a very unexpected property, since one would expect in principle merely some kind of monotonicity in time. We see also that for $\Gamma t>22$ the decay low is algebraic. 


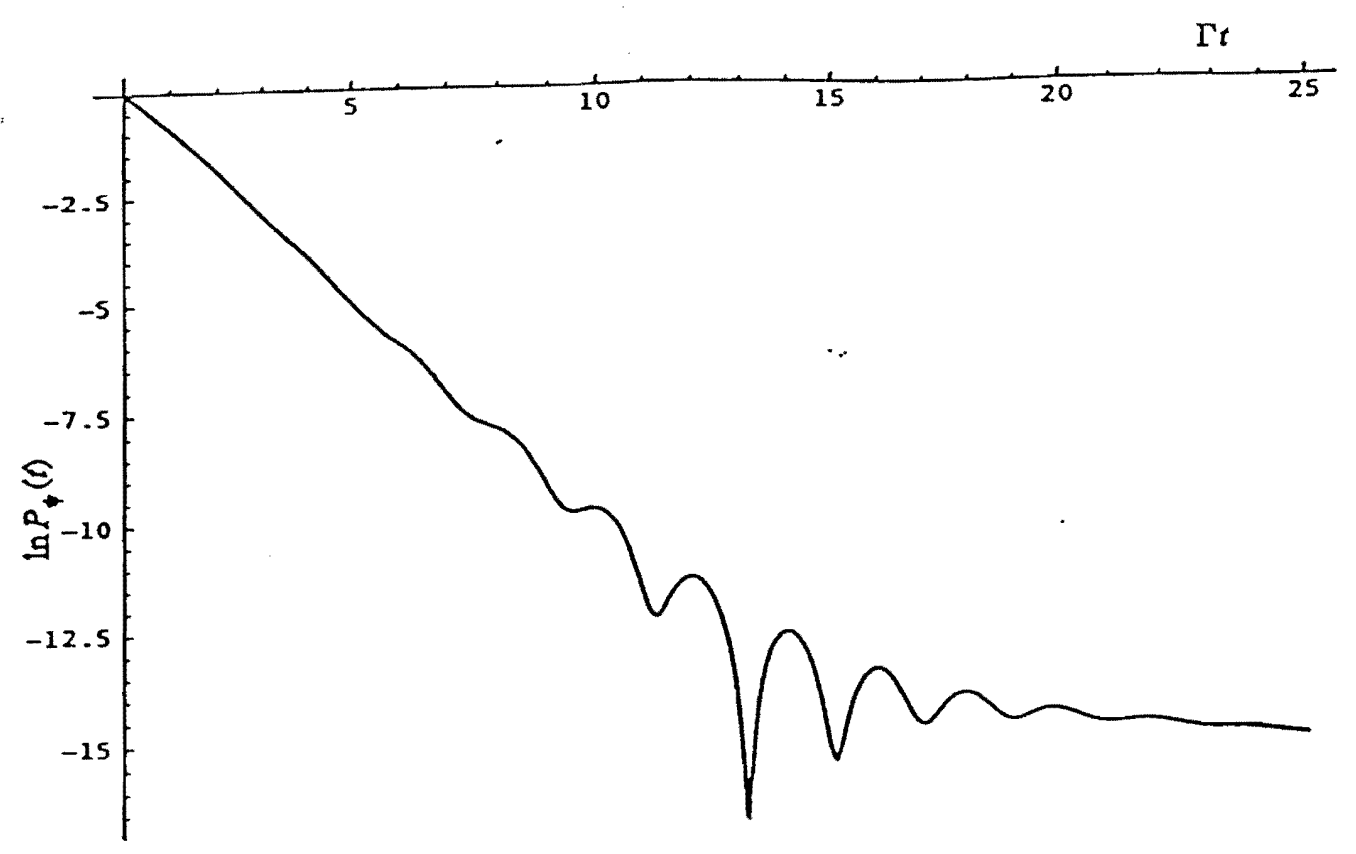

Fig. 2.1. The plot of $\ln \mathrm{P}_{\Psi}(\mathrm{t})$ versus $\Gamma \mathrm{t}$.

In order to illustrate better the deviation of the true decay law (2.4) from the exponential one we present in Fig.2.2 the plot of function $P_{y}(t) / \exp (-\Gamma t)$ as the function of $\Gamma \mathrm{t}$ in the case of $\rho$-meson.

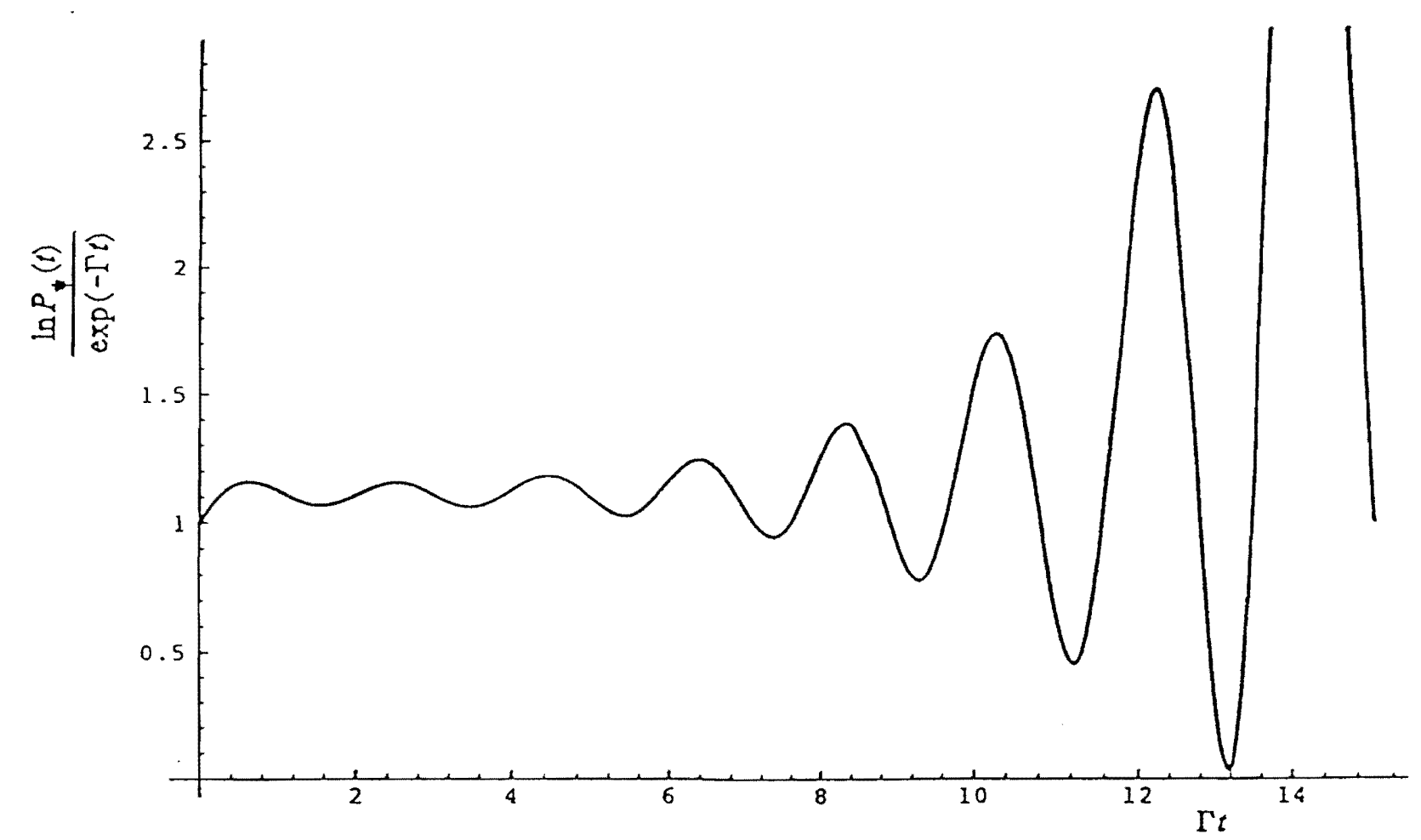

Fig. 2.2. The plot of $P_{\varphi}(t) / \exp (-\Gamma t)$ as the function of $\Gamma t$ for $\rho$-meson

We see that the ratio $\mathrm{P}_{\psi} / \exp (-\Gamma \mathrm{t})$ strongly oscillates with $\Gamma \mathrm{t}$ - the characteristic feature for all unstable particles, which will be more significant for unstable particles with a larger width $\Gamma$ or with the threshold energy $\lambda_{t r}$ close to $m$ as it is in case of $\phi$-meson decaying into $\mathrm{K} \overline{\mathrm{K}}$ pair. 
In most of analyses of mean life for an unstable elementary particle such as $\pi$, $\mathrm{K}, \rho, Z^{0}$ mesons or $n, \Lambda, \Sigma$ baryons one assumes that mean life $\tau_{\psi}^{\mathrm{m}}$ is connected with the decay width $\Gamma$ by the formula (1.1). This mean life would be obtained from the formula (2.10) if the decay law were pure exponential i.e. $P(t)=\exp (-\Gamma t)$. However we know from Thm 1.1 and (1.3) that pure exponential decay law is unphysical. Therefore in order to find the correct relation between mean life and decay law $\mathrm{P}_{\psi}(\mathrm{t})$ we have to use the general definition of $\tau_{\Psi}^{\mathrm{m}}$ given by Eq.(2.4). We first find $\tau_{\Psi}^{\mathrm{m}}$ for the energy distribution $g(\lambda)$ given by the Breit-Wigner formula (2.11). In this case $P_{\psi}(t)$ is given by (2.13). Inserting this expression into (2.4) we obtain:

$$
\begin{gathered}
\tau^{m}=\frac{1}{\Gamma}[1+\Delta \tau(\Gamma / \tilde{m})] \quad \text { where } \\
\Delta \tau_{\Psi}(\gamma)=N^{2}\left[1+\frac{1}{2 \pi} \frac{\gamma}{1+\frac{1}{4} \gamma^{2}}-\frac{1}{\pi} \operatorname{arctg} \frac{\gamma}{2}\right]-1 ; \gamma=\Gamma / \tilde{m}
\end{gathered}
$$

We see that in a realistic case of Breit-Wigner type of energy distribution the connection between $\tau_{\psi}, \tau_{\Psi}^{\mathrm{m}}$ and $\Gamma$ is given by a rather complex function of $\Gamma / \overline{\mathrm{m}}$. Hence from a theoretical point of view there is no justification in general to use for the life time or mean life the formula (1.1). It is noteworthy however that for small $\Gamma / \tilde{\mathrm{m}}$ from (2.19) we obtain:

$$
\tau_{\downarrow}^{m}(\Gamma / \tilde{m})=\frac{1}{\Gamma}\left[1+\frac{1}{\pi}\left(\frac{\Gamma}{\tilde{m}}\right)\right]+0\left[\left(\frac{\Gamma}{\tilde{m}}\right)^{2}\right]
$$

In order to illustrate the deviation of the true $\tau_{\Psi}^{\mathrm{m}}$ given by $(2.18)$ from ${ }_{\tau}^{\mathrm{m}}=\Gamma^{-1}$ we present in Fig. 2.3 the plot of $\tau_{\psi}^{\mathrm{m}} / \tau_{\Psi}^{\mathrm{m}}$ as the function of $\Gamma / \overline{\mathrm{m}}$.

We see that the $\tau_{\Psi}^{\mathrm{m}}$ deviates more significantly from $\dot{\tau}_{\Psi}^{\mathrm{m}}=\Gamma^{-1}$ if the ratio $\Gamma / \tilde{\mathrm{m}}$ or $\lambda_{\mathrm{tr}}$ increases. We note that for instance for the decay $\phi \rightarrow \mathrm{KK}, \lambda_{\mathrm{tr}}$ is close to $\mathrm{m}_{\phi}$ : hence $\Gamma / \tilde{\mathrm{m}}$ is in this case relatively large. 


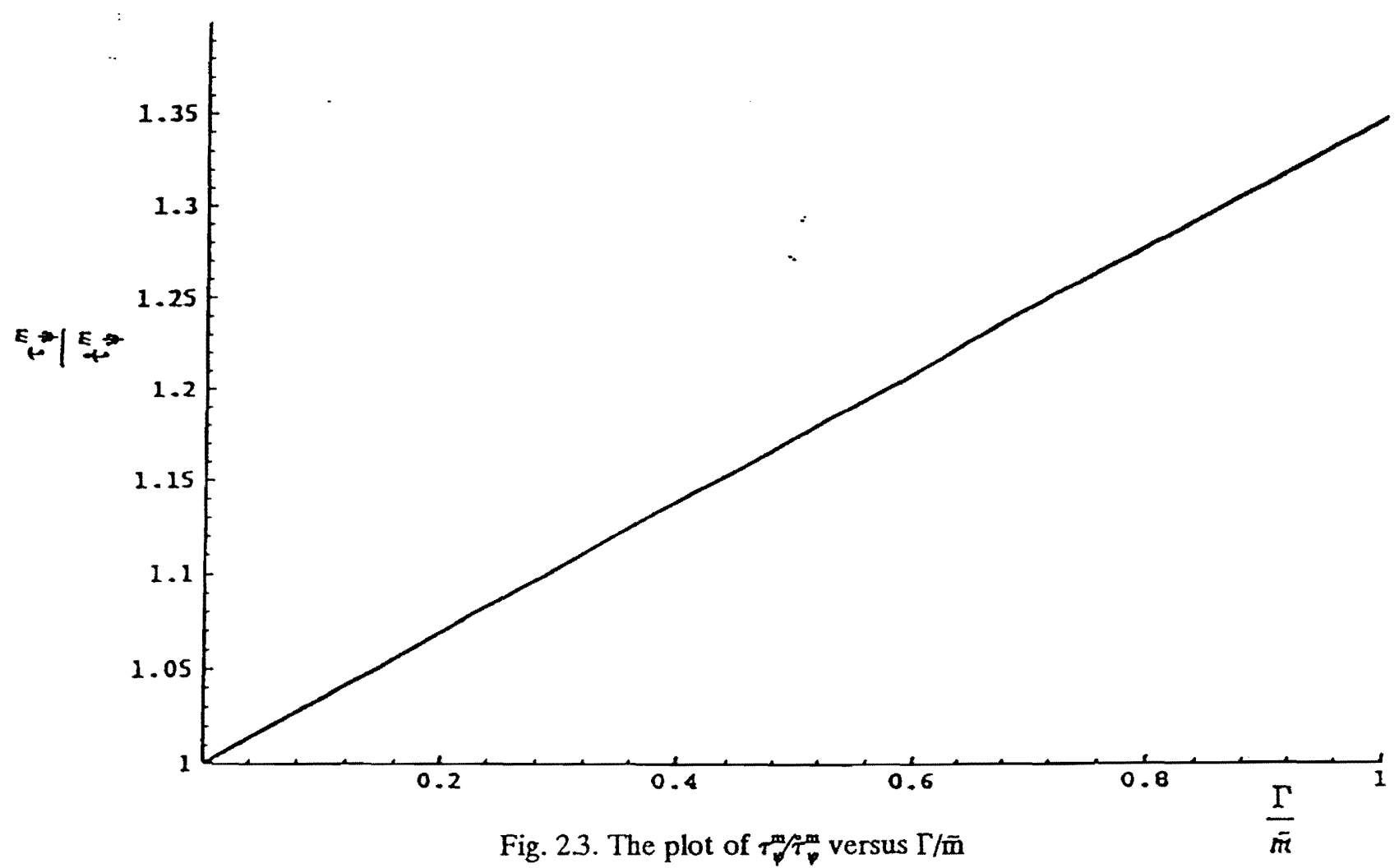

We give for comparison in Fig.2.4 the decay law $\mathrm{P}(\mathrm{t})$ for $\mathrm{Z}^{0}$-meson described by the B-W energy distribution.

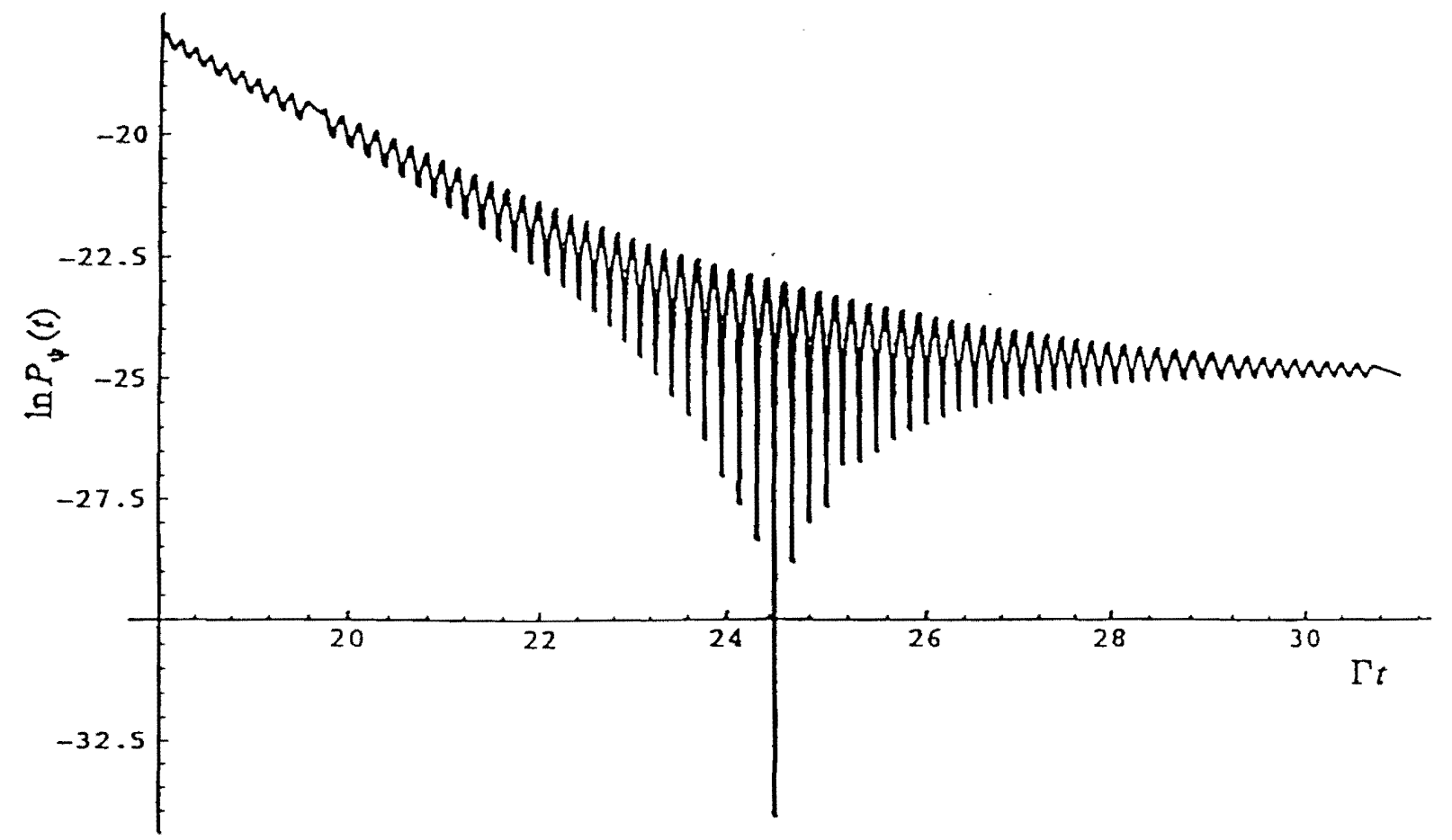

Fig. 2.4. The plot of $\ln P_{p}(t)$ versus $\Gamma t$ for $Z^{0}\left(\lambda_{t r}=0\right)$.

Comparing Fig. 2.4 and 2.1 we see that for $Z^{0}$-meson oscillations reach maximum for $\Gamma t c=24.47$ and the algebraic form of decay law dominates after $\Gamma t \simeq 27$. 


\section{Unstable Particles in Relativistic Quantum Field Theory}

We have described in Sec. 2 the properties of unstable particles using the BreitWigner energy distribution. The form of this distribution was derived in the nonrelativistic quantum mechanics [2]. It is interesting that the form of energy distribution derived in the frame-work of the relativistic quantum field theory is drastically different from the Breit-Wigner one. For instance Matthews and Salam have derived the following energy distribution [6]

$$
g(\lambda)=\frac{2 \Gamma N}{\pi} \frac{m \lambda \theta\left(\lambda-\lambda_{0}\right)}{\left(\lambda^{2}-m^{2}\right)^{2}+m^{2} \Gamma^{2}}
$$

where

$$
N=\left[\frac{1}{2}+\frac{1}{\pi} \operatorname{arctg}\left(\left(m^{2}-\lambda_{\pi}^{2}\right) / m \Gamma\right)\right]^{-1}
$$

Comparing (3.1) with (2.11) we see that, presently, the numerator is linearly dependent on energy whereas the denumerator is polynomial of fourth order in $\lambda$. Inserting (3.1) into (2.4) one obtains [2]

$$
v(t)=N \exp \left(-i S_{-} t\right)+\frac{i N}{2 \pi} \sum_{\alpha, \beta= \pm} \alpha \exp \left(i \beta s_{\alpha} t\right) E_{i}\left(-i \lambda_{\sigma} t-i \beta S_{\alpha} t\right)
$$

where

$$
S_{ \pm}=\left[\sqrt{\left(\sqrt{1+\gamma^{2}}+1\right) / 2} \pm i \sqrt{\left(\sqrt{1+\gamma^{2}}-1\right) / 2}\right] \Gamma / \gamma \quad \gamma=\Gamma / m
$$

For large $t$ we obtain

$$
v_{a s}=N \exp \left[-i S_{-}\right]+\frac{2 i N}{\pi t} \frac{m \lambda_{t r} \Gamma \exp \left(-i \lambda_{t} t\right)}{\left(m^{2}-\lambda_{t}\right)^{2}+m^{2} \Gamma^{2}}+R(t)
$$

where

$$
R(t)<\frac{4 N}{\pi\left(m-\lambda_{t r}\right)^{2} t^{2}}
$$


The asymptotic expansion for the decay law $\mathrm{P}(\mathrm{t})$ has the form

$$
\begin{aligned}
P(t) & =e^{-\mu t}+\frac{4 \Gamma^{2} \lambda_{t} \sin \left[t\left(\lambda_{t r}-\rho\right)\right]}{\pi \gamma\left[\left(\lambda_{t r}-\rho\right)^{2}+\mu^{2} / 4\right]\left[\left(\lambda_{t r}+\rho\right)^{2}+\mu^{2} / 4\right]} \cdot e^{-\mu t / 2} \cdot \frac{1}{t}+ \\
& +O\left(\frac{e^{-\mu / 2 \cdot t}}{t^{2}}\right)+\frac{4 \Gamma^{4} \lambda_{t r}^{2}}{\pi^{2} \gamma^{2}\left[\left(\lambda_{t r}-\rho\right)^{2}+\mu^{2} / 4\right]^{2}\left[\left(\lambda_{t r}+\rho\right)^{2}+\mu^{2} / 4\right]^{2}} \cdot \frac{1}{t^{2}}+O\left(\frac{1}{t^{4}}\right)
\end{aligned}
$$

where

$$
\begin{gathered}
\mu=\frac{2 \Gamma}{\gamma} \sqrt{\frac{\sqrt{1+\gamma^{2}}-1}{2}}=2 I m S_{-} \\
\rho=\frac{\Gamma}{\gamma} \sqrt{\frac{\sqrt{1+\gamma^{2}}+1}{2}}=\operatorname{Re} S_{-}
\end{gathered}
$$

and

We see that now even in the Region II for intermediate times the decay law is given by the formula

$$
P(t)=N^{2} \exp \left[-\Gamma t \cdot \frac{2}{\gamma} \sqrt{\frac{\sqrt{1+\gamma^{2}}-1}{2}}\right]
$$

which, for intermediate and large $\Gamma$, is significantly different from $\exp (-\Gamma \mathrm{t})$.

The formula for $\tau_{\psi}^{\mathrm{m}}$ for M-S energy distribution can be also written in the form (2.18) with $\Delta \tau(\Gamma / \tilde{\mathrm{m}})$ given by the formula

$$
\Delta \tau(\gamma)=\frac{2 N^{2}}{\pi} \int_{a}^{-} \frac{\sqrt{z \gamma+1}}{\left(1+z^{2}\right)^{2}} d z-1
$$

where

$$
a=\left[\left(\lambda_{t} / m\right)^{2}-1\right] / \gamma
$$

The integrals can be expressed by elementary functions, but the final formula is pretty complicated and will not be presented here.

We now give, for an illustration, two figures which describe the properties of the decay law $\mathrm{P}_{\psi}(\mathrm{t})$ and $\tau_{\psi}^{\mathrm{m}}$ in M-S theory. We take for comparison with figures 2.1-2.4 the same values for $\Gamma, \mathrm{m}$ and $\lambda_{\mathrm{tr}}$ and the same scale as in Sec. 2 . 
We give in Fig. 3.1 the plot of $\ln \mathrm{P}_{\phi}(\mathrm{t})$ for $\rho$-meson in order to illustrate the deviation from exponential law in M-S theory

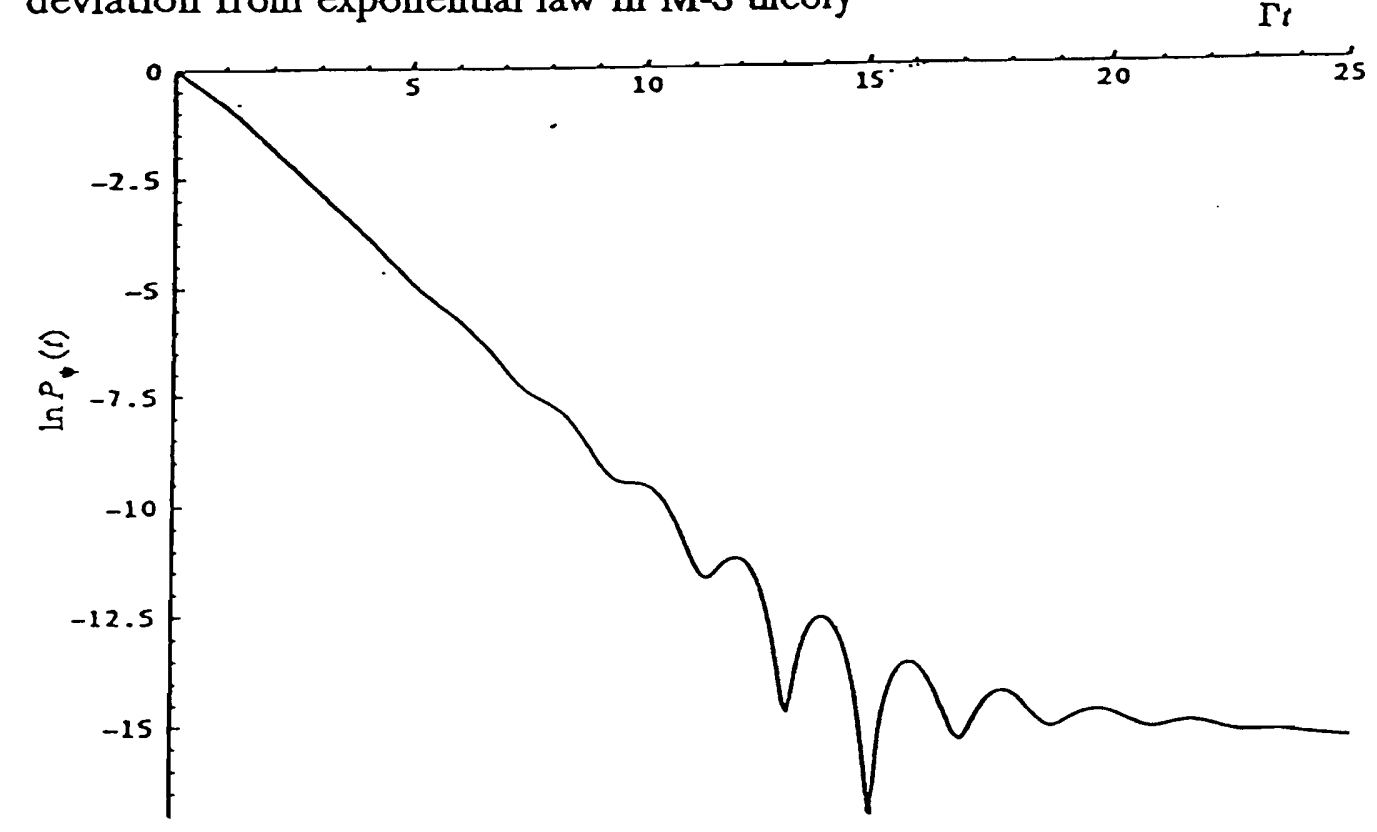

Fig. 3.1. The plot of $\operatorname{In} P_{\phi}(t)$ versus $\Gamma t$

Comparing the plots for $\ln \mathrm{P}_{\phi}(\mathrm{t})$ in Fig. 2.1 and Fig. 3.1 we see that in M-S theory the maximum of oscillations is shifted about two units in $\Gamma t$ variable but the shape of the curves are similar. We calculated the ratio $\mathrm{P}_{F}(\mathrm{t}) / \exp (-\Gamma \mathrm{t})$ and we also found that it was similar to the one corresponding to $\mathrm{B}-\mathrm{W}$ distribution.

We give below the plot of $\tau_{\psi}^{m} / \tau_{\psi}^{m}$ versus $\Gamma / m$ for various $\lambda_{\mathrm{t}}$.

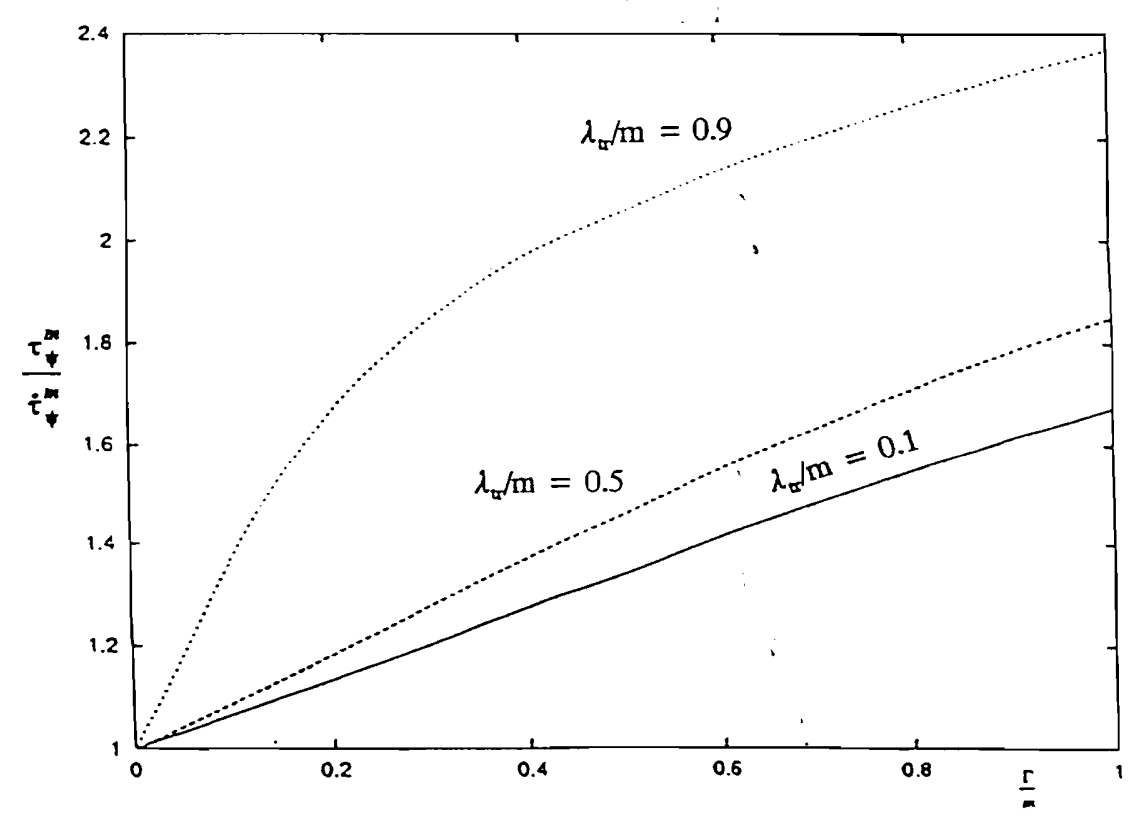

Fig. 3.2. The plot of $\tau_{\psi}^{\mathrm{m}} / \tau_{\psi}^{\mathrm{m}}$ versus $\Gamma / \mathrm{m}$ for various $\lambda_{\mathrm{tr}}$. 
Since the analytic dependence of $\tau_{\psi}^{\mathrm{m}}$ on $\lambda$ tr by (3.1) and (3.2) is different than in BW theory one obtains in the present case a rather strong dependence of $\tau_{\psi}^{\mathrm{m}}$ on $\lambda_{\mathrm{tr}}$.

We stress that the Figures 3.1 and 3.2 were obtained at the assumption that all unstable particles were produced at once at $t_{0}=0$. However in order to discuss a possibility to observe a non-exponential decay law, one must take into account the fact that we are not able to produce a reasonable number of unstable states at one instant of time (or during a negligibly short period). This fact is immaterial for a study of the exponential decay law, because the later satisfies:

$$
P\left(t+t_{0}\right)=P(t) P\left(t_{0}\right)
$$

If this relation is not satisfied, we observe that a sample of unstable states, "collected" during a finite time period will then decay according to some law different from $\mathrm{P}(\mathrm{t})$.

To see this let us assume that we have formed at some $-t^{\prime}$, cdt' unstable states. Then their number at some $t>t$ ' will be:

$$
d N(t)=c d t^{\prime} P\left(t-t^{\prime}\right)
$$

If we have been creating them at that constant rate $c$ between $-t_{0}$ and 0 , their number at $\mathrm{t}>0$ will be:

$$
N(t)=c \int_{-t_{0}}^{0} P\left(t-t^{\prime}\right) d t^{\prime}
$$

Let us define $P_{t_{0}}(t)$ as a decay law for a sample of states created with a constant rate between $-t_{0}$ and 0 and subjected to observation thereafter.

To have $P_{t_{0}}(t)$ we adjust $c$ as to have $N(0)=1$. If so:

$$
c=1 / \tau\left(t_{0}\right)=1 / \int_{0}^{t_{0}} P\left(t^{\prime}\right) d t^{\prime}
$$

and finally:

$$
P_{t_{0}}(t)=\frac{\tau\left(t_{0}+t\right)-\tau(t)}{\tau\left(t_{0}\right)}
$$

One can easily verify that if $P_{\psi}(t)=\exp (-\Gamma t)$ then $P_{t_{0}}(t)$ is also $\exp (-\Gamma t)$, what was to be expected. 
We could expect that this process of "collection" of a sample of unstable systems will "smooth out" fluctuations obsenwed an e.g. Figures 3.1. and 3.2. In fact, on Figures 3.3, 3.4 and 3.5 we can clearly observe this effect. It should be stressed, however, that some fluctuations seem to remain even after long "collection" periods.

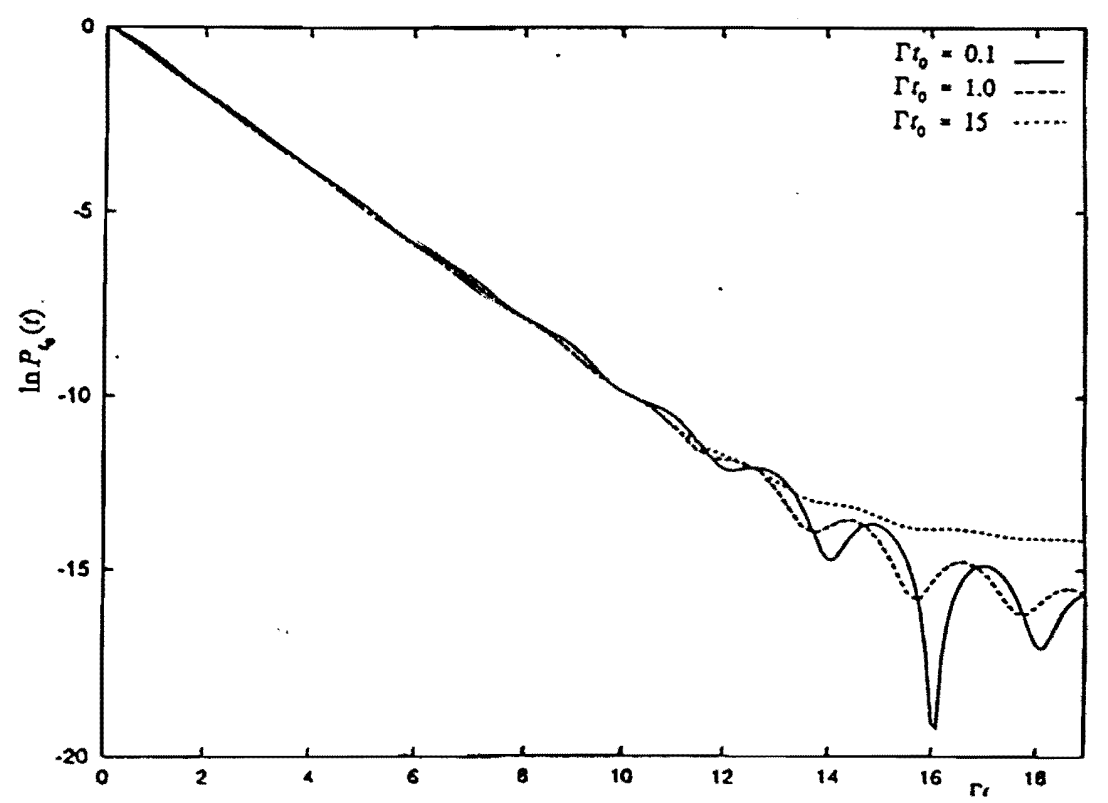

Fig. 3.3. The plot of $\ln \mathrm{P}_{\mathrm{t}_{\mathrm{o}}}(t)$ for various $\mathrm{t}_{\mathrm{o}}, \lambda_{\mathrm{v}} / \mathrm{m}=.1$ and $\gamma=.3$

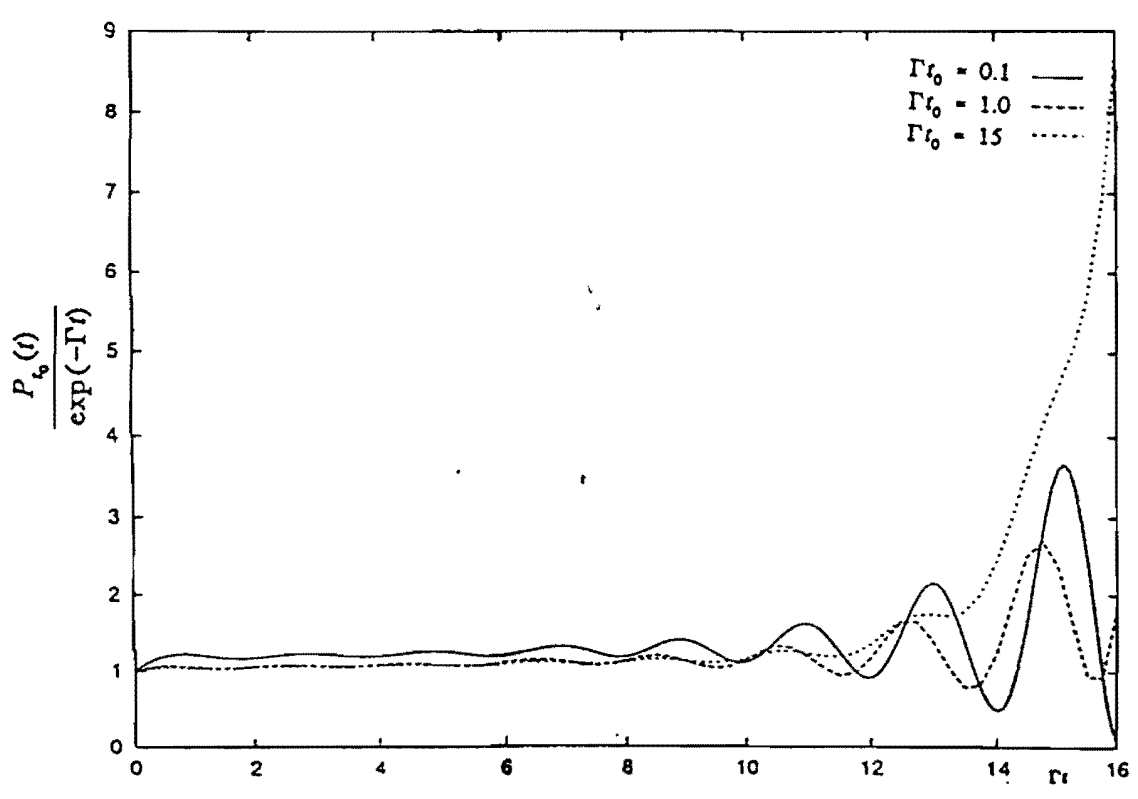

Fig. 3.4. The plot of $P_{t_{0}}(t) / \exp (-\Gamma t)$ for the same parameters as in Fig. 3.3 


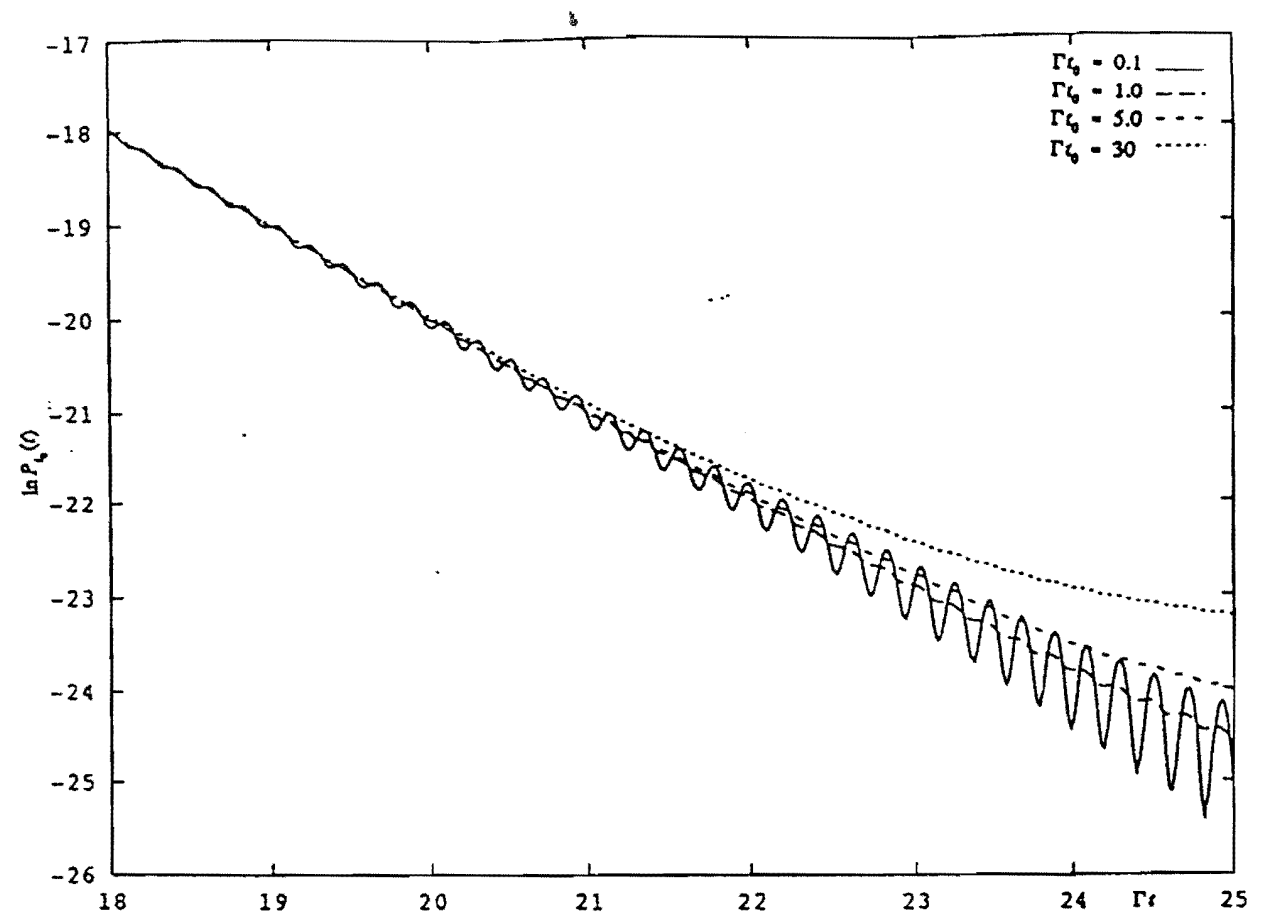

Fig. 3.5. The plot of $\ln \mathrm{P}_{\mathrm{f}}(\mathrm{t})$ for various $t_{\mathrm{o}}, \lambda_{\mathrm{t}} / \mathrm{m}=.1$ and $\gamma=.03$

We want to stress, once again, that in most realistic situations, particularity concerning rather long living unstable states, if would be rather $P_{\iota_{0}}(t)$ than $P_{\psi}(t)$ what would be comparable with experiment.

\section{Summary}

The commonly used decay law for an unstable state $\psi$

$$
P_{*}(t)=e^{-t / \tau^{m}}
$$

is, as known before, in contradiction with basic quantum properties of the state $\psi$.

Decay laws which follow from frequently used Breit-Wigner and Matthews-Salam energy distributions lead to oscillations of $\mathrm{P}_{\psi}(\mathrm{t})$ even for small and also for intermediate time scales where the exponential decay law could be treated as a satisfactory approximation to $\mathrm{P}_{\psi}(\mathrm{t})$.

However, it is only Matthews-Salam energy distribution which leads to $P_{\psi}(t)$ satisfying 


$$
\left.\frac{d P_{t}(t)}{d t}\right|_{t=0}=0
$$

what puts a question mark on a vallidity of the Breit-Wigner decay law.

Moreover a relation between a mean life of an unstable state $\psi, \tau^{\mathrm{m}}$ and $\Gamma$ - a width of an energy distrution of $\psi$, is not trivial and deviates definitely from $\Gamma=1 / \tau^{\mathrm{m}}$ characteristic for the exponential law.

In principle to determine $\tau$ for the exponential law it would suffice to carry out two measurements. To determine parameters of the decay laws following from BreisWigner and Matthews-Salam energy distributions one would need at last three and four measurements, correspondingly.

We observed that a problem of apparently unphysical oscillations of $\mathrm{P}_{\psi}(t)$ can be circumvented because of a necessity to collect sufficiently many unstable states to perform measurements on them. In such a case oscillations smooth out (if "collection times" are sufficiently large) and the basic feature - algebraic character of a decay law for large times, seems to be even more clear.

For small values of $\Gamma / \mathrm{m}$ (or $\Gamma / \tilde{\mathrm{m}}$ for the Breit-Wigner energy distribution) our decay laws differ, for intermediate tíme scales, only negligibly from the exponential one. Therefore to find significant deviations of $P_{\psi}(t)$ from the exponential decay law it is necessary to study such decays of unstable particles for which $\Gamma / \tilde{\mathrm{m}}$ is relatively large. The closer analysis indicates that the best candidate would be the $\phi$-meson decaying into $\mathrm{KK}$ pair or $\Lambda(1670)$ - resonance decaying into $\Lambda+\eta$. In both cases one should select the sample of the decay products for which the obtained mass of unstable particle would be the lowest i.e. on the extreme left side of mass distribution. In the next paper we shall discuss in detail the above and other proposals for the experimental verification of the deviation from the exponential decay law.

Finally we note that beside the B-W and M-S energy distribution there exists other energy distributions for unstable particles in a relativistic quantum field theory. For instance it would be interesting to investigate the decay law $\mathrm{P}_{\psi}(t)$ for energy distribution of unstable particle implied by analyticity and unitarity, as proposed by Renard [7]. 


\section{Acknowledgments}

The authors thank Prof. A.O. Barut, Prof. L. Fonda, Prof. G.C. Ghirardi and Prof. S. Wycech for inspiring discussions.

\section{REFERENCES}

1. See e.g. Review of Particle Properties, Phys.Rev. D45(1992) No.11.

2. P.Exner, "Open quantum systems and Feynman integrals", D.Reidel Dordrecht, 1984.

3. L.Fonda, G.C.Ghirardi and A.Rimini, Rep. on Progress in Phys. 41(1978)587 RPPHA

4. L.A.Khalfin, JETP 33 (1957) 1371 (in Russian) 00004

5. See e.g. J.Bailey et al., Nucl. Phys. B150(1979) 1

6. P.T.Matthews and A.Salam, Phys.Rev. 112(1958)283, ibid 115(1959)1079

7. F.M.Renard, Phys. Lett. 47B (1973)361 